\title{
ON THREE-DIMENSIONAL GENERALIZATIONS OF THE BOUSSINESQ AND KORTEWEG-DE VRIES EQUATIONS*
}

BY

\author{
E. INFELD
}

Institute of Nuclear Research, Warsaw

\begin{abstract}
Three-dimensional generalizations of two different forms of the Boussinesq equation are derived. They are investigated for stability of slowly varying nonlinear wavetrains. The results obtained are then compared with the stability properties following from the full water wave equations. Agreement is found to be good for $h_{0} k_{0}$ (depth times wavenumber) of order one. This is very satisfactory, as the Boussinesq equations are only supposed to be valid for small $h_{0} k_{0}$. In particular, one version of the Boussinesq equation is found to yield instability with respect to one-dimensional perturbations for $h_{0} k_{0}>1.5$ (as against 1.36 for the full equations). Finally, a similar comparison is performed for the three-dimensional Korteweg-de Vries equation.
\end{abstract}

1. Introduction. In this paper some model equations for water waves will be derived. They generalize existing model equations (Boussinesq and $\mathrm{K}-\mathrm{de} \mathrm{V}$ ) to three dimensions. These existing models were formulated in the late nineteenth century (1871 and 1895), the full equations for free surface waves being difficult to manipulate $[1,2]$.

We wish to investigate the free surface of an incompresssible fluid covering a flat bottom. The relevant equations are [3] (a suffix denotes partial differentiation):

$$
\begin{aligned}
& \mathbf{v}=(u, v, w)=\nabla \phi, \quad h=h_{0}+\eta(x, y, t), \\
& \nabla^{2} \phi=0, \\
& \eta_{t}+\phi_{x} \eta_{x}+\phi_{y} \eta_{y}-\phi_{z}=0
\end{aligned}
$$

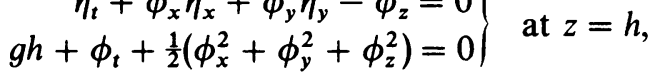

$$
\begin{aligned}
& \phi_{z}=0 \quad \text { at } z=0 \text {. }
\end{aligned}
$$

Here $h$ is the local depth, $h_{0}$ the average depth, $g$ the gravitational constant, $\mathbf{v}$ the fluid velocity and $z$ the distance from the bottom. The equation of the surface is $z=h(x, y, t)$ and the last three equations are boundary conditions; the first two on the surface (constraints following from the fact that fluid elements move with the surface; and pressure balance on the surface); and the third on the bottom (no motion perpendicular to the bottom). For more details see [3].

* Received April 3, 1979; revised version received September 10, 1979. The author would like to thank Drs. G. Rowlands, A. Kuszell and L. Turski for discussions that led to a better understanding of some aspects of the problem. 
Boussinesq looked at the two-dimensional problem $(\partial / \partial y=0)$ and found a much simpler set of equations in the small $h_{0} k_{0}$ limit. They are derived in Appendix 1 and take the form:

$$
\begin{gathered}
h_{t}+(u h)_{x}=0, \\
u_{t}+u u_{x}+g h_{x}+\frac{1}{3} c_{0}^{2} h_{0} h_{x x x}=0, \quad c_{0}^{2}=g h_{0} .
\end{gathered}
$$

Now $u$ is the average of $\phi_{x}$ over $z$ between 0 and $h$.

We will also investigate an alternate form of the Boussinesq equation. In the long wavelength limit (1.3) yields approximately

$$
\frac{D u}{d t}+g h_{x} \equiv u_{t}+u u_{x}+g h_{x} \approx 0 .
$$

We additionally assume

$$
\frac{\partial}{\partial x} \frac{D u}{d t} \approx \frac{D u}{d t}
$$

(in Appendix 1 a small parameter is introduced and $(D u / d x)_{x}-D u_{x} / d t$ is seen to be a higher order-term) and obtain

$$
g h_{x x} \approx \frac{-D u_{x}}{d t},
$$

leading to our second form of (1.5):

$$
u_{t}+u u_{x}+g h_{x}-\left(h_{0}^{2} / 3\right)\left(u_{x t}+u u_{x x}\right)_{x}=0 .
$$

This equation, together with (1.2), will constitute our alternate form of the Boussinesq equations. Both forms are Galilean-invariant, as is (1.1) (not all forms of Boussinesq found in the literature are). In Appendix 1 (1.2), (1.3), and (1.6) are derived more rigorously and generalized to three dimensions.

An equation similar to (1.2) and (1.6), but accurate to a higher order in the expansion parameter of Appendix 1, was derived by Su and Gardner [4].

When wave motion is further restricted to the positive $x$ direction we can finally obtain the Korteweg-de Vries equation

$$
u_{t}+u u_{x}+\left(c_{0} h_{0}^{2} / 6\right) u_{x x x}=0
$$

(Appendix 2).

Collecting the three models, we have:

BOUSSINESQ I (BI):

$$
\begin{aligned}
h_{t}+h u_{x} & =0, \\
u_{t}+u u_{x}+g h_{x}+\frac{1}{3} c_{0}^{2} h_{0} h_{x x x} & =0 .
\end{aligned}
$$

BOUSSINESQ II (BII):

$$
\begin{aligned}
h_{t}+(h u)_{x} & =0, \\
u_{t}+u u_{x}+g h_{x}-\frac{1}{3} h_{0}^{2}\left(u_{x t}+u u_{x x}\right) & =0 .
\end{aligned}
$$


KORTEWEG DE VRIES (K-de V):

$$
u_{t}+u u_{x}+\left(c_{0} h_{0} / 6\right) u_{x x x}=0 .
$$

2. Three-dimensional equations. When $\partial / \partial y$ is reinstated in (1.1) a three-dimensional situation is described. However, in all models under consideration the height $h$ is a function of $x, y$, and $t$ and so from now on we will speak of $(2+1)$-dimensional models. The $(2+1)$-dimensional extension of (1.7) and (1.9) is

$$
h_{t}+(h u)_{x}+(h v)_{y}=0
$$

whereas (1.8) and (1.10) are unaltered (Appendix 1). We are now one equation short, and to close the system we note from the existence of a velocity potential that

$$
v_{x}=u_{y} \text {. }
$$

Finally, in Appendix 2 the $(2+1)$-dimensional generalization of $(1.11)$ in the water wave context is shown to be

$$
u_{t}+u u_{x}+\frac{1}{6} u_{x x x}+\frac{1}{2} v_{y}=0, \quad v_{x}=u_{y}
$$

where $x$ is measured in units of $h_{0}$ and velocities in $c_{0}$. This equation has already been derived in the plasma physics context [5, 6], and in solid state theory [7].

In all these models $x$ and $y$ have uneven status. It is tacitly assumed that there will be a basic nonlinear structure moving in the $x$ direction, whereas the modulations will be in the $x, y$ plane.

All our model equations were derived in the long wavelength, shallow water limit $h_{0} k_{0} \rightarrow 0$. However, it would be useful to know for what values of the dimensionless parameter $h_{0} k_{0}$ these equations can be used. Sometimes model equations happen to be valid in regions that exceed what one has a right to expect from the derivation. This will in fact be seen to be the case here, especially for BI. To investigate our equations we will perform stability analyses for all three models and compare the results with those obtained from the full system (1.1) for arbitrary $h_{0} k_{0}$ [8].

3. Stability according to generalized Boussinesq II (BII). The original version of the Boussinesq equation entails some additional complications (Sec. 4), so we will rather perversely begin with a stability analysis for BII. It can be written as a system of firstand second-order differential equations:

BII:

$$
\begin{gathered}
h_{t}+(h u)_{x}+(h v)_{y}=0, \quad u_{t}+u u_{x}+h_{x}-P_{x}=0, \\
P=\frac{1}{3}\left(u_{x t}+u u_{x x}\right), \quad v_{x}=u_{y},
\end{gathered}
$$

where $x$ has been normalized to $h_{0}$ and velocities to $\left(g h_{0}\right)^{1 / 2}$ for convenience. We assume the existence of a stationary nonlinear wave and investigate it for stability with respect to small-amplitude, three-dimensional perturbations. This stationary wave propagates in the $x$ direction $(\partial / \partial y=0)$ and, due to Galilean invariance, we can use (3.1) with $\partial / \partial t=0$ to describe it. Thus in its own rest coordinate system the wave is given by

$$
\begin{aligned}
\frac{\partial h u}{\partial x} & =0, \quad \frac{\partial}{\partial x}\left(h+\frac{1}{2} u^{2}-P\right)=0, \\
P & =\frac{1}{3} u u_{x x} .
\end{aligned}
$$


The first two equations can be integrated:

$$
h u=u_{0}, \quad h_{0}=1, \quad h+\frac{1}{2} u^{2}-P=1+\frac{1}{2} u_{0}^{2},
$$

and these, together with the third, give one equation for $u$ :

$$
u_{x x}=3\left(u_{0} u^{-2}+\frac{1}{2} u-u^{-1}\left(1+\frac{1}{2} u_{0}^{2}\right)\right)
$$

(in general there will be two constants of integration, but they can be reduced to one constant $u_{0}$ by rescaling; see Appendix 3). We will look for nonlinear solutions to (3.4) in the form

$$
u=u_{0}+a \cos \sigma+O\left(a^{2}\right)
$$

where $a$ is small but finite. The first-order equations in $a$ yield

$$
\begin{aligned}
\sigma & =k_{0} x+\chi, \\
k_{0}^{2} & =3\left(u_{0}^{-2}-1\right), \quad u_{0}<1, \\
h_{1} & =-u_{0}^{-2} u_{1} .
\end{aligned}
$$

In second order

$$
\begin{aligned}
& u_{2}=\left(3 a^{2} / 4 k_{0}^{2}\right)\left(4 u_{0}^{-3}-u_{0}^{-1}\right)\left(1-\frac{1}{3} \cos 2 \sigma\right), \\
& h_{2}=\left(a^{2} / 4 u_{0}^{2} k_{0}^{2}\right)\left(\left[10 u_{0}-7\right] \cos 2 \sigma-3-6 u_{0}^{-2}\right) .
\end{aligned}
$$

The third-order expansion of (3.4) is of the form

$$
\left(\frac{d^{2}}{d x^{2}}+k_{0}^{2}\right) u_{3}=\text { terms involving } \cos \sigma+\text { nonres. }
$$

Thus secular terms will appear in $u_{3}$ unless we introduce many space variables and allow $\chi$ to be a function of $x_{2}$ [9]. This leads to the condition

$$
2 \frac{d \chi}{d x_{2}}=\left(3 a^{2} / 8 k_{0}\right)\left[3\left(6 u_{0}^{-4}-u_{0}^{-2}\right)+5\left(4 u_{0}^{-3}-u_{0}^{-1}\right)^{2} / k_{0}^{2}\right] .
$$

This concludes the calculation of the nonlinear wave structure.

We now linearize (3.1) around our nonlinear wave $u(x), h(x)$ :

$$
\begin{aligned}
& \frac{\partial}{\partial t} \delta h+\frac{\partial}{\partial x}(h \delta u+u \delta h)+\frac{\partial}{\partial y}(h \delta v)=0, \\
& \frac{\partial}{\partial t} u+\frac{\partial}{\partial x}(u \delta u+\delta h-\partial P)=0 \\
& \delta P=\frac{1}{3}\left[\frac{\partial^{2}}{\partial x \partial t} \delta u+u_{x x} \delta u+u \delta u_{x x}\right] \\
& \frac{\partial \delta u}{\partial y}=\frac{\partial \delta v}{\partial x}
\end{aligned}
$$

This is a system of equations in which the coefficients are periodic functions of $x$. We know from Floquet's theorem that solutions of the form

$$
P(x) e^{i(\mathbf{k} \cdot \mathbf{x}-\omega \boldsymbol{k})},
$$


where $P$ is periodic with the same period as $u$ and $h$, must exist. We will therefore take all perturbed quantities to be of this form and further assume $k$ and $\omega$ to be small quantities of order $a$ (this is equivalent to assuming slow variations of the nonlinear wave). Next we will look for resonances that arise in higher orders when the solutions up to a given order are multiplied by the coefficients of the equations. Physically, these resonances can be viewed as a wave-wave coupling effect involving two perturbed waves and the basic nonlinear wave. The procedure is well known $[10,11]$, and only an outline will be given here. Assume

$$
\begin{aligned}
\omega & =\omega_{1}+\omega_{2}+\cdots \quad \omega_{n} \sim a^{n-p} k^{p}, \\
\mathbf{k} & =\left(k_{x}, k_{y}\right)=k(\cos \theta, \sin \theta), \\
\delta u(x, t) & =e^{i(\mathbf{k} \cdot \mathbf{x}-\omega t)}\left[\delta u_{0}(x)+\delta u_{1}(x)+\cdots\right], \\
\delta h(x, t) & =e^{i(\mathbf{k} \cdot \mathbf{x}-\omega t)}\left[\delta h_{0}(x)+\delta h_{1}(x)+\cdots\right] .
\end{aligned}
$$

Eq. (3.8) is solved in lowest order by

$$
\delta u_{0}=A e^{i \sigma}+B e^{-i \sigma} \equiv \hat{A}+\hat{B}, \quad \delta v_{0}=0, \quad \delta h_{0}=-u_{0}^{-1} \delta u_{0} .
$$

The first-order form of (3.8) is

$$
\begin{gathered}
\frac{\partial}{\partial x}\left(\delta u_{1}+u_{0} \delta h_{1}\right)=i \omega_{1} \delta h_{0}-\frac{\partial}{\partial x}\left(h_{1} \delta u_{0}+u_{1} \delta h_{0}\right) \\
\frac{\partial}{\partial x}\left(u_{0} \delta u_{1}+\delta h_{1}-\delta P_{1}\right)=-\frac{\partial}{\partial x}\left(u_{1} \delta u_{0}\right)+i \omega_{1} \delta u_{0} \\
\delta P_{1}-\frac{1}{3} u_{0} \delta u_{1 x x}=\frac{1}{3}\left(i \omega_{1} \delta u_{0 x}+u_{1 x x} \delta u_{0}+u_{1} \delta u_{0 x x}+2 i k_{x} u_{0} \delta u_{0 x}\right) \\
\frac{d}{d x} \delta v_{1}=i k_{y} \delta u_{0}
\end{gathered}
$$

Secular terms will be avoided if

$$
\omega_{1}=k_{x} k_{0}^{2} u_{0}^{3} / 3
$$

and the first-order solutions are easily found to be

$$
\begin{aligned}
& \delta u_{1}=\left(3 / u_{0}^{2} k_{0}^{2}\right)\left(C_{1}-u_{0} C_{2}+\left[4 u_{0}^{-1}-u_{0}\right] X+u_{0}^{-2} k_{0}^{-2}\right)\left(u_{0}-4 u_{0}^{-1}\right)(\hat{A} \hat{\alpha}+B \hat{\beta}), \\
& \delta h_{1}=C_{1} / u_{0}-\left(\omega_{1} / u_{0}^{2} k_{0}\right)(\hat{A}-\hat{B})+2 u_{0}^{-2}(X+\hat{\alpha} \hat{A}+\hat{\beta} \hat{B})-u_{0}^{-1} \delta u_{1}, \\
& \delta v_{1}=\left(k_{y} / k_{0}\right)(\hat{A}-\hat{B})+C_{3},
\end{aligned}
$$

where $X=\alpha A+\beta B$ and $u_{1}=a \cos \sigma \equiv \hat{\alpha}+\hat{\beta}$. The constants $C_{1}, C_{2}$ and $C_{3}$ are obtained upon integrating (3.10), (3.11), and (3.13) respectively. To obtain the values of these constants we must write out the second-order versions of (3.10), (3.11), and (3.13) (Appendix 4) and again demand that secular terms vanish. The calculation is simplest for the second-order version of (3.13):

$$
\frac{\partial}{\partial x} \delta v_{2}=-i k_{x} \delta v_{1}+i k_{y} \delta u_{1}
$$


Upon averaging over a period we obtain, from the periodicity of $\delta v_{2}$,

$$
\left\langle\delta v_{1}\right\rangle=C_{3}=\tan \theta\left\langle\delta u_{1}\right\rangle=3 \tan \theta u_{0}^{-2} k_{0}^{-2}\left[C_{1}-u_{0} C_{2}+\left(4 u_{0}^{-1}-u_{0}\right) X\right] .
$$

The consistency conditions obtained from the second-order version of (3.10) and (3.11) are coupled and involve $\delta v_{1}$. With the aid of (3.15) they yield

$$
\begin{gathered}
C_{1}=\left[u_{0}^{-2}-2 u_{0}^{-3}-2 u_{0}+3\left(\tan ^{2} \theta\right) u_{0}^{-3} k_{0}^{-2}\left(1-4 u_{0}^{-2}\right)\right] X / \Delta, \\
C_{2}=\left(2+2 u_{0}^{-2}-u_{0}^{2}\right) X / \Delta, \quad \Delta=1+u_{0}^{2}+u_{0}^{-2}+3\left(\tan ^{2} \theta\right) k_{0}^{-2} u_{0}^{-4},
\end{gathered}
$$

and we are now in a position to write out the second-order equations with known right-hand sides (Appendix 4). If we add the first equation, multiplied by $-u_{0}^{-1}$ and integrated, to the integrated form of the second equation, and then the result to the third, we will obtain an equation for $u_{2}$ only, of the form

$$
\begin{aligned}
-\frac{1}{3} u_{0}\left(\frac{d^{2}}{d x^{2}}+k_{0}^{2}\right) \delta u_{2}= & \left(a_{11} A+a_{12} B\right) e^{i \sigma}+\left(a_{21} A+a_{22} B\right) e^{-i \sigma} \\
& + \text { terms proportional to } e^{i n}, \quad n \neq \pm 1 .
\end{aligned}
$$

This time round secular terms will only be avoided if $\operatorname{det}\left(a_{i j}\right)$ is zero. After a considerable amount of algebra this condition leads to a value for $\omega_{2}$. Combining the result with the known form of $\omega_{1}$, we obtain

$$
\begin{gathered}
\omega=\omega_{1}+\omega_{2}=\frac{1}{3} k_{0}^{2} u_{0}^{3} k \cos \theta \pm a u_{0} k \sqrt{\cos ^{2} \theta-u_{0}^{-4} k_{0}^{2} \sin ^{2} \theta} \sqrt{-2 a_{12}}, \\
a_{12}(\theta)=\frac{k_{0}^{2} u_{0}^{4}\left(4 u_{0}^{5}-25 u_{0}^{3}+9 u_{0}-13 u_{0}^{-1}-2 u_{0}^{-3}\right)+\tan ^{2} \theta\left(22 u_{0}^{-1}-11 u_{0}-2 u_{0}^{3}\right)}{u_{0}^{2}\left(1-u_{0}^{2}\right)\left(1+u_{0}^{2}+u_{0}^{-2}+3 \tan ^{2} \theta k_{0}^{-2} u_{0}^{-4}\right)} .
\end{gathered}
$$

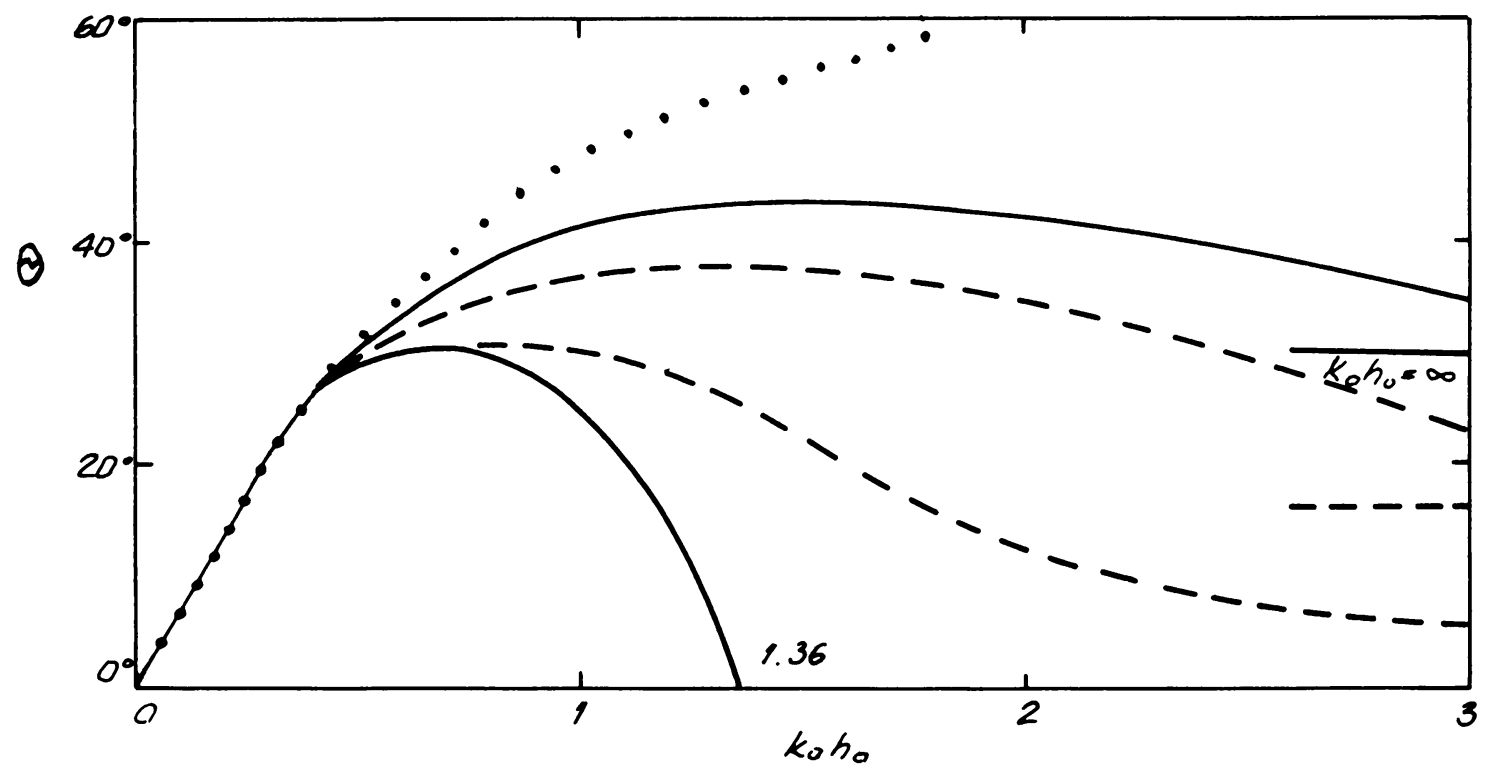

FIG. 1. Stability plot for water waves according to Hayes (solid lines) and BII (broken lines). Differences only become visible for $h_{0} k_{0}>\frac{1}{2}$, and serious for $h_{0} k_{0}>1$. Dots correspond to $\mathrm{K}$-de V. 
Thus the value of $\omega(\mathbf{k})$ splits in two, as usually happens in the presence of nonlinear waves $[12,13]$. Instability will set in between two critical angles $\theta$ given by

$$
\begin{aligned}
& \tan ^{2} \theta_{1}=k_{0}^{2} u_{0}^{4}, \\
& \tan ^{2} \theta_{2}=k_{0}^{2} u_{0}^{4} \frac{2 u_{0}^{-3}+13 u_{0}^{-1}-9 u_{0}+25 u_{0}^{3}-4 u_{0}^{5}}{3\left(22 u_{0}^{-1}-11 u_{0}-2 u_{0}^{3}\right)} .
\end{aligned}
$$

For $k_{0} \rightarrow 0, u_{0}^{2} \rightarrow 1$ and these angles merge. They can be drawn as functions of $k_{0}$ only, if (3.5) is used in (3.21). They are shown in Fig. 1 (where $h_{0}$ has been reinstated). The critical angles $\theta_{1}$ and $\theta_{2}$ are compared there with those obtained by Hayes from the full equations (1.1) [8]. Agreement is surprisingly good for $h_{0} k_{0}<1$ (after all, this was the expansion parameter!). The main difference between the two lower curves is that BII never gives instability for $\theta=0$ (one-dimensional perturbations), whereas the full set does when $h_{0} k_{0}>1.36$.

4. Stability according to generalized Boussinesq I (BI). Although BI looks simple enough, it furnishes two additional complications when an analysis similar to that of Sec. 3 is performed on it. These complications are most painlessly illustrated by taking the linearized forms of Eqs. (1.8), (2.1) and (2.2):

$$
\begin{aligned}
\frac{\partial \delta h}{\partial t}+\frac{\partial}{\partial x}(u \delta h+h \delta u)+\frac{\partial}{\partial y}(h \delta v) & =0, \\
\frac{\partial \delta u}{\partial t}+\frac{\partial}{\partial x}(u \delta u)+\frac{\partial \delta h}{\partial x}+\frac{1}{3} \frac{\partial^{3} h}{\partial x^{3}} & =0, \quad \frac{\partial \delta v}{\partial x}=\frac{\partial \delta u}{\partial y},
\end{aligned}
$$

and solving in the linear limit $a=0$. Now the perturbed quantities are simply proportional to $e^{i(\mathbf{k x}-\omega t)}$ and a linear dispersion relation is obtained. For $\theta=0$ this dispersion relation reduces to

$$
\left(\omega-k u_{0}\right)^{2}=k^{2}-\frac{1}{3} k^{4} .
$$

Thus $\omega \approx 0$ for the following $k$ :

$$
\begin{aligned}
& k \approx 0, \\
& k \approx \pm k_{0}, \quad k_{0}=\sqrt{3\left(1-u_{0}^{2}\right)} .
\end{aligned}
$$

Stationary waves (and thus weakly nonlinear waves) can only exist for $k_{0}<\sqrt{ } 3$. This implies that a drawing like Fig. 1 would become meaningless for $h_{0} k_{0}>\sqrt{ } 3$.

The second complication is a bit less trivial. By differentiating (4.2) we find that $\omega_{k}$ has three values for which $\omega=0$ :

$$
V_{1}=u_{0}+1, \quad V_{2}=-u_{0}+u_{0}^{-1}, \quad V_{3}=u_{0}-1
$$

(the second value $V_{2}$ is degenerate and corresponds to both $k=k_{0}$ and $k=-k_{0}$ ). For one particular value of $u_{0}\left(=\frac{1}{2}\right), V_{1}=V_{2}$ and this presents a new possibility of wave-wave coupling between the stationary modes

$$
k= \pm k_{0}, \quad \omega=0,
$$

and the modes

$$
\omega \approx V_{1} k, \quad \omega \approx V_{2}\left(k \pm k_{0}\right) .
$$


This new mode-mode coupling mechanism will in general lead to a third critical angle $\theta_{3}$ (from the above we expect this angle to be zero for $u_{0}=\frac{1}{2}$ ).

A calculation similar to that of Sec. 3 yields

$$
\begin{aligned}
\tan ^{2} \theta_{1} & =\left(2 u_{0}^{2}+1\right)\left(1-u_{0}^{2}\right) u_{0}^{-4}, \\
\tan ^{2} \theta_{2} & =\frac{\left(23 u_{0}^{4}-4 u_{0}^{2}-10 u_{0}^{6}\right)\left(1-u_{0}^{2}\right)}{\left(2 u_{0}^{8}+5 u_{0}^{6}-4 u_{0}^{4}\right)}, \\
\tan ^{2} \theta_{3} & =\left(u_{0}^{-2}-4\right)\left(1-u_{0}^{2}\right), \quad u_{0}^{2} \leq 1 .
\end{aligned}
$$

Again, these angles can be obtained in terms of $k_{0} h_{0}$ only. For $h_{0} k_{0}<1$ they look much like those for the other two models (Hayes and BII; again $\theta_{1}$ and $\theta_{2}$ merge and give the right slope at zero). However, the lower stable region will now be bounded on the right by $\theta_{3}$ and will be topologically like Hayes' case. The critical value of $h_{0} k_{0}$ is 1.5 , as can be seen from (4.10) and (4.4). This is not very different from Hayes' value 1.36 (actually known before Hayes $[10,14])$. For larger values of $h_{0} k_{0}$, however, the model breaks down and finally becomes meaningless for $h_{0} k_{0}>\sqrt{ } 3$.

5. Three-dimensional $\mathbf{K}$-de $\mathbf{V}$. In Appendix 2 the three-dimensional $\mathrm{K}$-de $\mathrm{V}$ equation is derived for water waves. An analysis similar to that of Sec. 3 for this equation has already been performed in the plasma physics context [13]. The dispersion relation is, in our notation,

$$
\omega=\frac{1}{3} h_{0}^{2} k_{0}^{2} \pm \frac{a h_{0} k_{0}}{2 \sqrt{ } 3} \sqrt{\left(h_{0}^{2} k_{0}^{2}-\tan ^{2} \theta\right)^{2} /\left(h_{0}^{2} k_{0}^{2}+\tan ^{2} \theta\right)} k \cos \theta .
$$

(This equation was obtained from Eq. (7.4) of the reference with the substitution $\omega / k_{x} \rightarrow$ $\alpha \omega / k_{x}, \tan ^{2} \theta \rightarrow \alpha \tan ^{2} \theta, \alpha=2 \sqrt{ } 3 /\left(h_{0} k_{0}\right)^{2}$. This substitution is sanctioned by the fact that if $u(x, y, t)$ and $v(x, y, t)$ solve (2.3), so will $\alpha u\left(\alpha^{-1 / 2} x, \alpha^{-1} y, \alpha^{-3 / 2} t\right)$ and $\alpha^{3 / 2} v\left(\alpha^{-1 / 2} x\right.$, $\left.\alpha^{-1} y, \alpha^{-3 / 2} t\right)$.)

Thus $\theta_{1}=\theta_{2}=\tan ^{-1}\left(h_{0} k_{0}\right)$ and a single, degenerate curve with the right slope at zero is obtained (dotted line in Fig. 1). However, the instability region for small $h_{0} k_{0}$ is in any case confined to an extremely narrow solid angle. Thus the fact that the width has shrunk to zero for the $\mathrm{K}$-de $\mathrm{V}$ equation is perhaps not surprising.

A comparison of all three models-BI, BII, and $\mathrm{K}$-de V-with the full equations shows that whereas the Boussinesq models offer an excellent picture of the stability problem for weakly nonlinear waves when $h_{0} k_{0}<1, \mathrm{~K}$-de $\mathrm{V}$ is somewhat simplified and certainly limited to $h_{0} k_{0}<\frac{1}{2}$.

6. Summary. Generalized $(2+1)$-dimensional forms of the Boussinesq equations are seen to furnish very good models for the modulations of weakly nonlinear water waves for $h_{0} k_{0}<1$. A $(2+1)$-dimensional extension of the Korteweg-de Vries equation, on the other hand, gives a somewhat simplified theory, less exact than Boussinesq, but still qualitatively good for $h_{0} k_{0}<\frac{1}{2}$.

Note added in proof: A one-dimensional analysis of the Su-Gardner equation, mentioned in Sec. 1 and derived in [4], yields stability for all $h_{0} k_{0}$. In this respect it is thus seen to be no better than our BII. 


\section{REFERENCES}

[1] J. Boussinesq, Comptes Rendus 12, 755 (1871)

[2] D. J. Korteweg and G. de Vries, Phil. Mag. (5) 39, 422 (1895)

[3] G. B. Whitham, Linear and nonlinear waves, John Wiley, 1974

[4] C. H. Su and C. S. Gardner, J. Math. Phys. 10, 536 (1969)

[5] B. B. Kadomtsev and V. I. Pitvyashvili, Dokl. Akad. Nauk SSSR 192, 757 (1970)

[6] M. Kako and G. Rowlands, Plasma Phys. 18, 165 (1976)

[7] I. A. Kunin, Teoria uprugyh sryed s mikrostrukturoy, Izdat. Nauka, Moskva, 1975

[8] W. D. Hayes, Proc. R. Soc. Lond. A332, 199 (1973)

[9] N. N. Bogolyubov and Y. A. Mitropolski, Asymptotic methods in the theory of nonlinear oscillations, Hindustani, Delhi, 1961

[10] T. B. Benjamin, Proc. R. Soc. Lond. A299, 59 (1967)

[11] C. H. Su, Phys. Fluids 13, 1275 (1970)

[12] G. B. Whitham, Proc. R. Soc. Lond. A283, 238 (1965)

[13] E. Infeld and G. Rowlands, Proc. R. Soc. Lond. A366, 537 (1979)

[14] G. B. Whitham, J. Fluid Mech. 27, 399 (1967)

[15] E. Infeld, G. Rowlands and M. Hen, Acta Phys. Polon. A54, 131 (1978)

Appendix 1. For convenience we normalize all lengths to $h_{0}$ and velocities to $\left(g h_{0}\right)^{1 / 2}$. Then $(1.1)$ takes the form

$$
\begin{array}{cc}
\mathrm{v}=(u, v, w)=\nabla \phi, & \nabla^{2} \phi=0, \\
\eta_{t}+\phi_{x} \eta_{x}+\phi_{y} \eta_{y}-\phi_{z}=0 & \text { at } z=1+\eta, \\
\eta+\phi_{t}+\frac{1}{2}\left(\phi_{x}^{2}+\phi_{y}^{2}+\phi_{z}^{2}\right)=0 & \\
\phi_{z}=0 & \text { at } z=0 .
\end{array}
$$

We next stretch the coordinates $x, y, t$ according to the scheme

$$
\xi=\varepsilon^{1 / 2} x, \quad \rho=\varepsilon y, \quad \tau=\varepsilon^{1 / 2} t,
$$

and assume amplitudes of waves and/or solitons to be small and of the form

$$
\begin{aligned}
& \eta=\varepsilon \eta^{(1)}+\varepsilon^{2} \eta^{(2)}+\cdots \\
& \phi=\varepsilon^{1 / 2} \phi^{(1)}+\varepsilon^{3 / 2} \phi^{(2)}+\cdots
\end{aligned}
$$

Eqs. (A1.1), (A1.6), etc. imply

$$
\begin{aligned}
& u=\varepsilon u^{(1)}+\varepsilon^{2} u^{(2)}+\cdots, \\
& v=\varepsilon^{3 / 2} v^{(1)}+\varepsilon^{5 / 2} v^{(2)}+\cdots
\end{aligned}
$$

The general solution to (A1.2) satisfying (A1.5) is, in the new system,

$$
\phi=f+\sum_{m=1}^{\infty}(-)^{m} \frac{z^{2 m}}{(2 m) !}\left[\varepsilon \frac{\partial^{2}}{\partial \xi^{2}}+\varepsilon^{2} \frac{\partial^{2}}{\partial \rho^{2}}\right]^{m} f .
$$

So $\operatorname{far} f(x, y, t)$ is arbitrary. Upon differentiation of (A1.8) by $x$ we obtain

$$
\phi_{x}=u-\frac{\varepsilon}{2} z^{2} u_{x x}+O\left(\varepsilon^{2}\right) .
$$


We must also satisfy (A1.3) and (A1.4). Retaining terms up to and including $\varepsilon^{5 / 2}$, these two conditions imply

$$
\begin{gathered}
\eta_{\tau}^{(1)}+w_{\xi}^{(1)}+\varepsilon\left(\eta_{\tau}^{(2)}+w_{\xi}^{(2)}+\left[\eta^{(1)} w^{(1)}\right]_{\xi}-\frac{1}{6} w_{\xi \xi \xi}^{(1)}+v_{\rho}^{(1)}\right)+O\left(\varepsilon^{2}\right)=0, \\
w_{\tau}^{(1)}+\eta_{\xi}^{(1)}+\varepsilon\left(w_{\tau}^{(2)}+w^{(1)} w_{\xi}^{(1)}-\frac{1}{2} w_{\xi \tau \tau}^{(1)}+\eta_{\xi}^{(2)}\right)+O\left(\varepsilon^{2}\right)=0, \\
w=f_{x} .
\end{gathered}
$$

Averaging (A1.9) over the depth yields

$$
w=\bar{u}+\frac{\varepsilon}{6} \bar{u}_{x x}+O\left(\varepsilon^{2}\right)
$$

whereas it will be sufficient to take

$$
v=\bar{v}+O(\varepsilon)
$$

When (A1.11) and (A1.12) are used in (A1.10) and we go back to the original variables, we obtain after some manipulations

$$
h_{t}+(h \bar{u})_{x}+(h \bar{v})_{y}=0, \quad \bar{u}_{t}+\bar{u} \bar{u}_{x}+h_{x}+\frac{1}{3} h_{x x x}=0 .
$$

Finally, to lowest order from (A1.1),

$$
\bar{v}_{x}=\bar{u}_{y}
$$

This completes the derivation of BI.

The expression

$$
\frac{\partial}{\partial x} \frac{D u}{d t}-\frac{D u_{x}}{d t}=u_{x}^{2}=\varepsilon^{3} u_{\xi}^{(1)}+o\left(\varepsilon^{3}\right)
$$

is of third order and so can be neglected. Eq. (A1.13) is thus equivalent to (1.9) and (1.10) under this expansion scheme. (Actually, the unwanted term (A1.14) would enter (1.10) differentiated by $x$ and thus would be of order $\frac{7}{2}$, a full $\varepsilon$ above all significant terms.)

Appendix 2. We now assume that the basic nonlinear wave or soliton propagates in the positive $x$ direction with a constant velocity near one $\left(\left(g h_{0}\right)^{1 / 2}\right.$ before renormalization). We stretch the coordinates according to the scheme

$$
\xi=\varepsilon^{1 / 2}(x-t), \quad \tau=\varepsilon^{3 / 2} t
$$

and all other quantities as in Appendix 1. Once again (A1.8) is obtained. Conditions (A1.3) and (A1.4) both give the same equation to lowest order in $\varepsilon$ :

$$
\eta^{(1)}=u^{(1)}=f_{\xi} .
$$

To close the system we must go to next order:

$$
\begin{array}{r}
\eta_{\tau}^{(1)}-\eta_{\xi}^{(2)}+u_{\xi}^{(2)}+2 u^{(1)} u_{\xi}^{(1)}-\frac{1}{6} u_{\xi \xi \xi}^{(1)}+v_{\rho}^{(1)}=0, \\
u_{\tau}^{(1)}-u_{\xi}^{(2)}+\eta_{\xi}^{(2)}+u^{(1)} u_{\xi}^{(1)}+\frac{1}{2} u_{\xi \xi \xi}^{(1)}=0 .
\end{array}
$$

Adding these two equations we obtain, in view of (A2.1),

$$
u_{\tau}^{(1)}+\frac{3}{2} u^{(1)} u_{\xi}^{(1)}+\frac{1}{6} u_{\xi \xi \xi}^{(1)}+\frac{1}{2} v_{\rho}^{(1)}=0 .
$$


Finally,

$$
v_{\xi}^{(1)}=u_{\rho}^{(1)}
$$

is again obtained from the identity $\phi_{x y}=\phi_{y x}$.

Thus we have obtained the three-dimensional $\mathrm{K}$-de $\mathrm{V}$ equation (KadomtsevPitvyashvili equation) as a $(2+1)$-dimensional model for shallow water waves. Complete agreement with (2.3) could be obtained via a trivial renormalization.

Appendix 3. A more general form of the integrated equations in (3.3) would be

$$
h u=u_{0}, \quad h+\frac{1}{2} u^{2}-P=T .
$$

However, the transformation $h=\alpha h^{\prime}, u=\alpha^{1 / 2} u^{\prime}, P=\alpha P^{\prime}, u_{0}=\alpha^{3 / 2} u_{0}^{\prime}$, where $\alpha^{1 / 2}$ is the positive root of

$$
u_{0} X^{5}+X^{2}-T
$$

will lead to

$$
h^{\prime} u^{\prime}=u_{0}^{\prime}, \quad h^{\prime}+\frac{1}{2} u^{\prime 2}-P^{\prime}=1+\frac{1}{2} u_{0}^{\prime 2} .
$$

To preserve the form of the remaining equations (3.1) we would need the further transformations $x=x^{\prime}, t=\alpha^{-1 / 2} t^{\prime}, v=\alpha^{1 / 2} v^{\prime}$.

Appendix 4. The second-order equations are:

$$
\begin{aligned}
& \frac{\partial}{\partial x}\left(\delta u_{2}+u_{0} \delta h_{2}\right)= \frac{-\partial}{\partial x}\left(h_{2} \delta u_{0}+u_{2} \delta h_{0}+h_{1} \delta u_{1}+u_{1} \delta h_{1}\right) \\
&- i k_{x}\left(\delta u_{1}+h_{1} \delta u_{0}+u_{0} \delta h_{1}+u_{1} \delta h_{0}\right) \\
&- i k_{y} \delta v_{1}+i \omega_{1} \delta h_{1}+i \omega_{2} \delta h_{2} \\
& \frac{\partial}{\partial x}\left(u_{0} \delta u_{2}+\delta h_{2}-\delta P_{2}\right)= \frac{-\partial}{\partial x}\left(u_{2} \delta u_{0}+u_{1} \delta u_{1}\right) \\
&-i k_{x}\left(u_{0} \delta u_{1}+u_{1} \delta u_{0}+\delta h_{1}-\delta P_{1}\right) \\
&+i \omega_{1} \delta u_{1}+i \omega_{2} \delta u_{0} \\
& \delta P_{2}-\frac{1}{3} u_{0} \delta u_{2 x x}=\frac{1}{3}\left[-i \omega_{1} \delta u_{1 x}-i \omega_{2} \delta u_{0 x}+k_{x} \omega_{1} \delta u_{0}+\delta u_{0} \delta u_{2 x x}\right. \\
&+\delta u_{1} u_{1 x x}+u_{1} \delta u_{1 x x}+u_{2} \delta u_{0 x x}+2 u_{0} \delta u_{0 x x_{2}}-u_{0} k_{x}^{2} \delta u_{0} \\
&\left.+2 i k_{x} u_{0} \delta u_{1 x}+2 i k_{x} u_{1} \delta u_{0 x}\right]
\end{aligned}
$$

